\title{
Médiévales
}

Langues, Textes, Histoire

77 | automne 2019

Mathématiques. Savoirs et enseignements (OrientOccident)

\section{Pourquoi et comment étudier l'histoire de l'enseignement des mathématiques dans les sociétés islamiques entre 750 et 1500}

Why and How to Study the History of Mathematical Education in Islamic

Societies between 750 and 1500

\section{Sonja Brentjes}

Traducteur : Marc Moyon et Christopher Lucken

\section{(Q) OpenEdition}

\section{Journals}

Édition électronique

URL : https://journals.openedition.org/medievales/10194

DOI : 10.4000/medievales. 10194

ISSN : $1777-5892$

Éditeur

Presses universitaires de Vincennes

Édition imprimée

Date de publication : 5 décembre 2019

Pagination : 11-35

ISBN : 978-2-37924-061-4

ISSN : 0751-2708

\section{Référence électronique}

Sonja Brentjes, « Pourquoi et comment étudier l'histoire de l'enseignement des mathématiques dans les sociétés islamiques entre 750 et 1500 », Médiévales [En ligne], 77 | automne 2019, mis en ligne le 01 janvier 2022, consulté le 22 avril 2022. URL : http://journals.openedition.org/medievales/10194 ; DOI : https://doi.org/10.4000/medievales.10194 
Sonja Brentjes

\section{Pourquoi et comment étudier l'histoire de l'enseignement des mathématiques dans les sociétés islamiques entre 750 et 1500}

J'interroge dans cette étude la façon dont a été abordée l'histoire de l'enseignement des mathématiques dans les sociétés islamiques. Je me concentre sur la période comprise entre 750 et 1500 , parce que les sources disponibles pour ce type d'étude historiographique changent dans une certaine mesure de nature et de caractère avec l'arrivée des trois « Empires de la poudre à canon » (les Ottomans, les Safavides et les Moghols), changement qui reflète d'importantes modifications concernant le statut institutionnel et le contenu de l'éducation, mais aussi les défis provoqués par l'arrivée d'ambassadeurs, de missionnaires et d'autres types de voyageurs des pays d'Europe catholiques et protestants à partir du début du XVI e siècle.

Pour comprendre mes positions méthodologiques, il faut tenir compte des présupposés et des débats historiographiques qui ont dominé pendant les cinq ou six dernières décennies l'histoire des mathématiques et des sciences exactes (comme on a l'habitude de les appeler). C'est pourquoi je commencerai par rappeler certains éléments de ces débats afin de clarifier, d'une part, les limites que j'ai tenté de franchir en enquêtant avec la plus grande attention possible sur l'histoire de l'enseignement des mathématiques dans les sociétés islamiques, et, d'autre part, les positions que j'ai dû remettre en question, voire abandonner. Comme je l'explique ci-dessous, certaines des opinions admises parmi les historiens des mathématiques et des sciences exactes dans les sociétés islamiques ont généré de sérieux obstacles à une telle histoire.

Alors qu'elle avait privilégié les seuls traités, comprenant de nouveaux résultats et de nouvelles méthodes, la communauté scientifique a manifesté au cours des dix ou vingt dernières années davantage d'intérêt pour les textes élémentaires. Les recherches se sont surtout concentrées sur des questions de contenu, laissant de côté les autres aspects de ces textes : leur apparence matérielle et leur organisation formelle, ainsi que les 
questions que posent leur usage, leur statut social, leur valeur économique et leur valeur culturelle. C'est pourquoi j'expose dans la deuxième section de cette étude mon point de vue sur ce que nous pouvons gagner lorsque nous adoptons une approche large et contextualisée pour étudier l'histoire de l'enseignement des mathématiques. J'associe cette explication à la présentation d'un certain nombre de questions qui, à mon avis, doivent être abordées si l'on veut produire une histoire de l'enseignement des mathématiques.

La troisième section se concentre sur l'un des débats historiographiques passés qui a constitué un obstacle particulièrement important à une étude sérieuse de l'enseignement des mathématiques et de ses différents cadres institutionnels. On en est venu à penser principalement l'histoire institutionnelle de l'éducation dans les pays d'Islam selon un modèle de conflits et d'exclusion. Les effets néfastes d'une telle conception pour une approche holistique de l'histoire de l'éducation dans les sociétés islamiques continuent d'avoir un impact regrettable sur les perspectives des collègues, à l'intérieur comme à l'extérieur de l'histoire des mathématiques et des sciences exactes dans ces sociétés. Je présente un certain nombre d'enseignements que l'on peut tirer de l'étude de diverses sources qui remettent en question ce modèle où dominent le conflit et la marginalisation.

Les quatrième et cinquième sections abordent deux problèmes méthodologiques concernant la manière dont peut s'élaborer une histoire fiable de l'enseignement des mathématiques. La première porte sur la façon de s'y prendre pour faire en sorte que les traités mathématiques et les écrits apparentés parlent de leur objectif et de leur contexte. La seconde cherche à identifier les autres sources historiques qui pourraient être explorées de manière significative afin de replacer dans leurs contextes spécifiques, en temps et en lieu, les textes mathématiques et scientifiques qui nous sont parvenus. Je discute ainsi, à titre d'exemples, des certificats d'enseignement, des éléments paratextuels et des dictionnaires biographiques.

Dans ma conclusion, enfin, je mets l'accent sur les principaux résultats que j'ai acquis au cours des deux dernières décennies en étudiant un matériel qui s'avère pertinent pour l'histoire de l'enseignement des mathématiques dans des villes et à des périodes particulières ${ }^{1}$.

1. Mes principales publications développant mon point de vue sur le sujet sont : S. BRENTJES, «On the Location of the Ancient or "Rational" Sciences in Muslim Educational Landscapes (AH 500-1100) », Bulletin of the Royal Institute for Inter-Faith Studies, 4/1 (2002), p. 47-71 ; «Reflections on the Role of the Exact Sciences in Islamic Culture and Education between the Twelfth and the Fifteenth Centuries », dans M. AвATTouy éd., Études d'histoire des sciences arabes, Casablanca, 2007, p. 15-33 ; " The Study of Geometry According to al-Sakhāwī (Cairo, 15th c.) and al-Muhibbī (Damascus, 17th c.) », dans J. W. Dauben, S. Kirschner, A. Kühne, P. Kunitzsch et R. Lorch éd., Mathematics Celestial and Terrestrial. Festschrift for Menso Folkerts zum 65. Geburtstag, Halle (Saale), 2008, p.323-341 ; «Euclid's Elements, Courtly Patronage and Princely Education », Iranian Studies, 


\section{Débats historiographiques des années 1960 à 2010}

En ce qui concerne les sciences mathématiques et les sciences exactes, l'approche historiographique de la seconde moitié $\mathrm{du} \mathrm{xx}^{\mathrm{e}}$ siècle s'est concentrée dans une large mesure sur la période du califat abbasside (7501258) et sur leurs contemporains, de la péninsule Ibérique à l'Afghanistan, avec quelques excursions dans des périodes historiques plus tardives (avec en particulier des études sur la théorie planétaire dans l'Empire Ilkhanide [1256-1335], à Maragha et Tabriz, et d'autres sur les recherches effectuées à la madrasa et à l'observatoire de Samarkand sous le patronage du prince Timuride et, plus tard, du chef de famille Ulugh Beg [m. 1449]). Les sciences mathématiques qui ont été étudiées sont les différents domaines de l'astronomie, la géométrie théorique, la théorie des nombres, l'optique, l'algèbre, les systèmes de calcul et certaines parties de la technologie ou de la mécanique (automates, machines de levage à eau, balances). Toujours sous la rubrique des sciences exactes, l'astrologie a été ajoutée à cet éventail de connaissances disciplinaires. Le principal objectif de ces recherches était de découvrir des résultats novateurs. Les questions relatives à l'enseignement et celles portant sur le contexte historique servaient au mieux de brèves informations biographiques, lorsqu'elles étaient abordées.

Cette focalisation sur de nouveaux résultats au détriment d'autres programmes de recherche a été principalement motivée par des intérêts politiques et professionnels des historiens issus de divers pays des trois blocs politiques - l'Occident, les pays socialistes et les pays dits « du Tiers Monde »- ainsi que par les positions alors généralement dominantes en matière de recherche dans l'histoire des mathématiques et des sciences en général (approches internalistes contre approches externalistes ${ }^{2}$ ). Les

41 (2008), p. 441-463 ; « The Mathematical Sciences in the Safavid Empire : Questions and Perspectives », dans D. Hermann et F. SPeziale éd., Muslim Cultures in the Indo-Iranian World during the Early-Modern and Modern Periods, Berlin, 2010, p. 325-402 ; « Teaching the Mathematical Sciences in Islamic Societies. Eighth-Seventeenth Centuries », dans A. KarP et G. Schubring éd., Handbook on the History of Mathematics Education, New York, 2014, p. 85-107 ; « Safavid Art, Science and Courtly Education », dans N. Sidoli et G. VAN Brummelen éd., From Alexandria, Through Baghdad. Surveys and Studies in the Ancient Greek and Medieval Islamic Mathematical Sciences in Honor of J. L. Berggren, Heidelberg, 2014, p. 489-504 ; " Teaching the Sciences in Ninth-Century Baghdad as a Question in the History of the Book : The Case of Abū Yūsuf Ya'qūb b. Isḥāq al-Kindī (d. after 256/870) », Intellectual History of the Islamicate World, 5/1-2 (2016), p. 1-27; « Science, Religion, and Education », dans S. Brentjes, T. Edis et L. Richter-Bernburg éd., 1001 Distortions. How (Not) to Narrate the History of Science, Medicine and Technology in Non-Western Cultures, Würzburg, 2016, p. 133-150 ; Teaching and Learning the Sciences in Islamicate Societies (800-1700), Turnhout, 2018.

2. Les internalistes estiment que l'histoire d'une science donnée doit être expliquée principalement à partir de ses structures de connaissances internes (concepts, arguments, méthodes). Selon les externalistes, le contexte social dans lequel une science s'est développée a joué un rôle essentiel, sinon primordial. 
historiens des mathématiques et des sciences exactes en pays d'Islam étaient en très grande majorité internalistes. Ils ont réalisé d'importantes découvertes $^{3}$. Les résultats auxquels ils sont arrivés montrent cependant qu'il est souhaitable, et même nécessaire, que les études sur les textes et les instruments prennent en compte leurs contextes. Mais leur principal objectif était de prouver que les érudits des pays d'Islam étaient hautement qualifiés et qu'ils étaient donc capables d'obtenir de nouveaux résultats, de développer de nouvelles méthodes, de nouvelles techniques et de nouveaux modèles, ou même de rivaliser avec les héros ancestraux de la traditionnelle histoire des sciences en Occident, en particulier Ptolémée et Aristote.

Deux approches méthodologiques ont dominé ce type d'études : (1) la comparaison de ces innovations avec celles d'auteurs anciens ou avec celles d'érudits travaillant dans l'Europe latine médiévale ; (2) la traduction des éléments novateurs dans le ou les langages des mathématiques modernes ( $\mathrm{XIX}^{\mathrm{e}}-\mathrm{XX}^{\mathrm{e}}$ siècles). La première approche privilégie une analyse diachronique, soit une enquête qui situe un texte (ou un instrument) dans une chaîne temporelle composée d'autres textes (ou instruments), situés dans le passé ou dans le futur par rapport au texte (ou à l'instrument) étudié. Le présent de l'objet étudié (texte ou instrument) est considéré comme une composante majeure de ces recherches pour autant qu'il existe des objets comparables. Des éléments contemporains de nature substantiellement différente n'ont été que rarement, sinon jamais, considérés comme utiles dans l'analyse d'un tel objet (texte ou instrument).

3. (1) Les modèles non ptolémaïques des mouvements planétaires ; (2) la solution des problèmes liés aux devoirs religieux fondamentaux des musulmans (la direction de la prière, les heures de prière, le début d'un nouveau mois) par des procédures géométriques ou arithmétiques - exactes ou approximatives - formulées dans des textes ou des instruments dans un contexte soit géographique, soit astronomique ; (3) la construction de nouveaux types d'astrolabes, quadrants, compas ou instruments composés ; (4) la formulation et la démonstration (réussie ou non) de théorèmes relevant de la théorie des nombres, de la géométrie et de l'optique ; (5) des solutions technologiques pour l'irrigation et les machines de pompage qui leur sont associées ; (6) la reconstitution de cadres disciplinaires et leur contenu en optique ou en astronomie ; (7) l'identification de communautés scientifiques régionales dont les membres entretiennent d'importantes relations et possèdent les mêmes méthodes, les mêmes techniques et les mêmes ouvrages de référence, par exemple dans la péninsule Ibérique et l'Afrique du Nord, le Yémen rasulide, l'Égypte mamelouke et la Syrie ou dans les capitales et les centres des provinces ottomanes. Voir J. L. BERGGREN, « History of Mathematics : The Present State of the Art », Middle East Studies Association Bulletin, 19 (1985), p. 9-33, repris dans N. Sidoli et G. Van Brummelen éd., From Alexandria, Through Baghdad..., p. 51-71 ; J. L. BergGREN, « Mathematics and Her Sisters in Medieval Islam : A Selective Review of Work Done from 1985 to 1995 », Historia Mathematica, 24 (1997), p. 407-440, repris dans N. Sidoli et G. Van Brummelen éd., From Alexandria, Through Baghdad..., p. 73-99 ; G. VAN BRUmmelen, "A Survey of Research in the Mathematical Sciences in Medieval Islam from 1997 to 2011 », dans N. Sidoli et G. VAN BRUmmelen éd., From Alexandria, Through Baghdad..., p. 101-138. 
L'arrière-plan politique et idéologique de l'accent mis sur l'innovation a favorisé une orientation historiographique tournée vers l'Antiquité, en particulier vers les textes grecs, vers l'Europe du Moyen Âge et, parfois, vers les temps modernes, principalement dans les centres universitaires en France, en Angleterre, en Italie et, parfois, aux Pays-Bas. Alors que le cadre comparatif de la première approche devait son existence à des conflits politiques, idéologiques, académiques, et à des positions prises par des universitaires en France, en Allemagne, en Italie et dans une moindre mesure en Grande-Bretagne ou aux Pays-Bas au cours du XIX ${ }^{\mathrm{e}}$ siècle, la seconde approche était principalement une création de mathématiciens devenus historiens des mathématiques au $\mathrm{xx}^{\mathrm{e}}$ siècle $^{4}$. Avec leurs partisans, ils ont poussé plus loin la comparaison diachronique en direction de la Renaissance et, même, de l'époque moderne (de l'Europe occidentale).

$\mathrm{La}$ possibilité d'exprimer des problèmes mathématiques et leur traitement dans des langages différents, mais mathématiquement équivalents, a encouragé les interprétations qui identifient les travaux des érudits des $\mathrm{IX}^{\mathrm{e}}, \mathrm{X}^{\mathrm{e}}$ ou $\mathrm{XI}^{\mathrm{e}}$ siècles avec les travaux des érudits du XVII ${ }^{\mathrm{e}}$ au $\mathrm{XIX}^{\mathrm{e}}$ siècles. On a soutenu que des savants des pays d'Islam avaient déjà formulé des résultats appartenant au calcul infinitésimal, aux théories des fonctions, aux théories algébriques modernes, ou qu'ils avaient ouvert un nouveau champ disciplinaire qui ne sera connu de l'Europe qu'à partir du $\mathrm{XIX}^{\mathrm{e}}$ siècle, comme la géométrie algébrique ${ }^{5}$. Pour les mathématiciens, de telles identifications peuvent être et sont souvent significatives. D'un point de vue historique, cependant, elles induisent en erreur et entravent les efforts visant à comprendre les travaux des érudits des pays d'Islam selon leurs propres termes et dans leur propre monde intellectuel.

4. F. CHARETtE, Orientalisme et histoire des sciences : L'historiographie européenne des sciences islamiques et hindoues, 1784-1900, thèse de M.A., Université de Montréal, 1995 ; J. Peiffer, « France », dans J. W. Dauben et C. J. SCRIBA éd., Writing the History of Mathematics : Its Historical Development, Bâle/Boston/Berlin, 2002, p. 3-43 (voir, en particulier, "Oriental Studies and the History of Mathematics in the Nineteenth Century », p. 14-20) ; S. BrentJeS, «Practicing History of Mathematics in Islamicate Societies in 19thCentury Germany and France », dans V. R. Remmert, M. R. Schneider et H. K. Sørensen éd., Historiography of Mathematics in the 19th and 20th Century, Cham, 2016, p. 25-52 ; O. Neugebauer, The Exact Sciences in Antiquity, Providence, 1957 ; B. L. van Der Waerden, A History of Algebra von al-Khwarizmi to Emmy Noether, Berlin/Heidelberg, 1985 ; A. P. JuschKewITsCh, Geschichte der Mathematik im Mittelalter, Leipzig, 1964 ; B. A. RozenFeLD, A History of Non-Euclidean Geometry : Evolution of the Concept of a Geometric Space, New York, 1988.

5. A. P. Juschкewitsch, Geschichte der Mathematik im Mittelalter, Leipzig, 1964. Parmi les nombreux travaux de Roshdi Rashed où se trouvent de telles affirmations, deux seulement sont choisis ici à titre d'exemples : R. RASHED, Histoire des sciences arabes, t. 2, Mathématiques et physique, Paris, 2003 ; D'al-Khwarizmi à Descartes. Études sur l'histoire des mathématiques classiques, Paris, 2011. 
Les deux approches reposent sur deux affirmations : (1) celle, explicite, que les savants du passé doivent être valorisés ; (2) celle, rarement admise, qu'une telle valorisation ne peut être réalisée qu'en plaçant les savants des pays d'Islam au moins au même niveau que - et de préférence à un niveau supérieur à - celui des savants grecs anciens ou des sociétés européennes chrétiennes ou laïques de toute époque. Ces deux désirs dévalorisent alors l'étude d'éléments - en premier lieu ceux produits pour et dans les écoles qui, par leur contenu et leur contexte, ne peuvent contribuer à la réalisation des objectifs plus nobles que sont l'innovation et les grandes réalisations.

Les deux approches dominantes ont toutefois eu des conséquences inattendues. Les résultats impressionnants obtenus par chacune d'entre elles ont favorisé de nouvelles discussions à propos de problèmes historiographiques qui avaient déjà été formulés par des historiens des mathématiques, des astronomes, des arabisants et par leurs interlocuteurs universitaires au XIX ${ }^{e}$ siècle. Les principaux conflits historiographiques du $\mathrm{XIX}^{\mathrm{e}}$ siècle ont porté sur la question des relations entre les réalisations de la Grèce antique et les contributions dites arabes aux sciences mathématiques. Les « Arabes » méritent-ils le respect parce qu'ils « ont conservé des textes mathématiques grecs en traduction arabe », bien qu'ils étaient par ailleurs improductifs, ou ont-ils apporté des résultats scientifiques significatifs au progrès général des sciences ? En raison de graves erreurs dans la compréhension du langage et du contenu d'un texte astronomique par Abū 1-Wafā' al-Būzjānī (940-998), la deuxième position a été éliminée de ce débat dans les dernières décennies du Xix ${ }^{\mathrm{e}}$ siècle. En outre, de nouvelles discussions et de nouvelles perspectives portant sur des problèmes courants ont été ajoutées au cours du $\mathrm{xx}^{\mathrm{e}}$ siècle par les historiens s'intéressant de manière plus générale aux pays d'Islam. Contesté au XIX siècle uniquement par le réformateur musulman Jamāl al-Dīn al-Afghānī (1838-1897), le verdict raciste, antisémite et historiquement faux prononcé en 1883 par Ernest Renan (1823-1892) sur la mort de la philosophie après le décès d'Ibn Rushd en 1198, sur la persécution générale et sur l'extinction définitive des sciences en pays d'Islam, fut accepté pour toutes les disciplines considérées par les historiens modernistes comme des sciences ${ }^{6}$. Les deux points de

6. E. Renan, L'Islamisme et la Science, Paris, 1883. Renan déclare dans son discours à la Sorbonne, par exemple : « Toute personne un peu instruite des choses de notre temps voit clairement l'infériorité actuelle des pays musulmans, la décadence des États gouvernés par l'islam, la nullité intellectuelle des races qui tiennent uniquement de cette religion leur culture et leur éducation. Tous ceux qui ont été en Orient ou en Afrique sont frappés de ce qu'a de fatalement borné l'esprit d'un vrai croyant, de cette espèce de cercle de fer qui entoure sa tête, la rend absolument fermée à la science, incapable de rien apprendre ni de s'ouvrir à aucune idée nouvelle. [...] Persuadé que Dieu donne la fortune et le pouvoir à qui bon lui semble, sans tenir compte de l'instruction ni du mérite personnel, le musulman a le plus profond mépris pour l'instruction, pour la science, pour tout ce qui constitue l'esprit européen. [...] Bientôt la race turque prendra l'hégémonie de l'islam, et fera prévaloir partout son manque total 
vue ont gouverné les perspectives, les interprétations et les objectifs de la recherche jusqu'à la première décennie $\mathrm{du} \mathrm{XxI}^{\mathrm{e}}$ siècle. De nouvelles positions sont apparues au cours du $\mathrm{Xx}^{\mathrm{e}}$ siècle, qui insistent sur l'hostilité profonde et durable de l'ensemble de la classe des érudits religieux à l'égard des sciences anciennes, sur la séparation nette entre les disciplines religieuses et philologiques d'une part et les sciences anciennes d'autre part, sur l'impact destructeur du Tahāfut al-falāsifa (L'incohérence des philosophes) d'Abū Hāmid al-Ghazālī (1058-1111) et sur l'accroissement continu de la superstition.

Trois thèses interprétatives furent adoptées par toute l'histoire intellectuelle des sociétés islamiques qui ont fortement influencé les intérêts manifestés par la recherche et le choix de ses objets particuliers : (1) le déclin ${ }^{7}$; (2) l'opposition entre ce que l'on a appelé l'orthodoxie islamique et les sciences anciennes ${ }^{8}$; (3) la marginalisation des sciences mathématiques,

d'esprit philosophique et scientifique. À partir de ce moment, à quelques rares exceptions près, comme Ibn-Khaldoun, l'islam ne comptera plus aucun esprit large ; il a tué la science et la philosophie dans son sein. [...] L'arabe, qui se prête si bien à la poésie et à une certaine éloquence, est un instrument fort incommode pour la métaphysique. Les philosophes et les savants arabes sont en général d'assez mauvais écrivains. [...] L'islamisme, en réalité, a donc toujours persécuté la science et la philosophie. Il a fini par les étouffer. [...] Dans la première période, l'islam, miné par les sectes et tempéré par une espèce de protestantisme (ce qu'on appelle le motazélisme), est bien moins organisé et moins fanatique qu'il ne l'a été dans le second âge, quand il est tombé entre les mains des races tartares et berbères, races lourdes, brutales et sans esprit. [...] L'islam, c'est l'union indiscernable du spirituel et du temporel, c'est le règne d'un dogme, c'est la chaîne la plus lourde que l'humanité ait jamais portée. [...] Il y a en Asie des éléments de barbarie analogues à ceux qui ont formé les premières années musulmanes et ces grands cyclones d'Attila, de Gengiskhan. Mais la science leur barre le chemin. Si Omar, si Gengiskhan avaient rencontré devant eux une bonne artillerie, ils n'eussent pas dépassé les limites de leur désert [...] » (p. 2-3, 14-17 et 23). Pour la réponse d'al-Afghani, voir N. KedDIE, An Islamic Response to Imperialism : Political and Religious Writings of Sayyid Jamal al-Din al-Afghani, Berkeley (CA), 1968.

7. Ce terme est commun à de nombreuses discussions sur les développements ou les stagnations dans les sociétés islamiques. Il ne s'applique pas seulement à un domaine particulier de la culture ou à une discipline mathématique. La domination généralisée de ce concept historiographique et son manque de pertinence ont été discutés dans S. vON Hees éd., Inhitat - The Decline Paradigm : Its Influence and Persistence in Writing Arab Cultural History, Würzburg, 2017. Ce terme a également profondément imprégné les idées des historiens des mathématiques, des sciences, de la médecine, de la technologie et de la philosophie dans les sociétés islamiques : voir S. BRENTJes, « The Prison of Categories "Decline" and Its Company », dans F. Opwis et D. ReIsman éd., Islamic Philosophy, Science, Culture, and Religion. Studies in Honor of Dimitri Gutas, Leyde/Boston, 2012, p. 131-155. Alors que les historiens de la philosophie ont désormais abandonné ce terme considéré comme improductif, les historiens des mathématiques continuent à l'utiliser.

8. Le document de base qui a donné le ton à ces discussions a été rédigé en 1915 par l'éminent chercheur hongrois Ignaz Goldziher (1850-1921). Je me réfère ici à la traduction anglaise : I. GoldzIHER, "The Attitude of the Old Islamic Orthodoxy Toward the "Ancient Sciences" », dans M. Swartz éd., Studies in Islam, New York, 1981, 185-215. J'y reviendrai plus loin. 
de la philosophie, de la médecine et des sciences occultes après le soidisant «âge d'or », en particulier dans les établissements d'enseignement, mais aussi dans les cours ${ }^{9}$. Un exposé de ces trois thèses se retrouve dans quantité d'études ${ }^{10}$.

Ces trois thèses ont contribué à privilégier l'étude des contenus mathématiques de haut niveau durant ce qu'on appelle l' «âge d'or ». Elles ont constitué de véritables obstacles à une plus grande exploration du contexte et des conditions dans lesquels se déroulaient les pratiques mathématiques, y compris en matière d'éducation ${ }^{11}$. Elles ont également découragé toute contribution systématique sur des thèmes mathématiquement pertinents dans les siècles qui ont suivi l' « âge d'or », à trois exceptions majeures près cependant : (1) la construction d'instruments scientifiques ; (2) la théorie planétaire ; (3) la discipline astronomique de calcul des heures, qui n'a émergé comme ensemble organisé de connaissances disciplinaires et socialement reconnues qu'après la destruction du califat abbasside par les Mongols en $1258^{12}$.

9. Le principal livre affirmant cela - de manière plutôt stricte et rigide - pour les établissements d'enseignement a été rédigé par G. MAKDISI (1920-2002) : The Rise of Colleges. Institutions of Learning in Islam and the West, Édimbourg, 1981.

10. A. I. SABRA, «The Appropriation and Subsequent Naturalization of Greek Science in Medieval Islam », History of Science, 25 (1987), p. 223-243, reproduit dans F. J. RAGEP, S. P. RageP et S. Livesey éd., Tradition, Transmission, Transformation. Proceedings of Two Conferences held at the University of Oklahoma, Leyde-New York-Cologne, 1996, p. 3-28 ; A. I. SABRA, « Situating Arabic Science : Locality versus Essence », Isis, 87 (1996), p. 654-670, repris dans M. H. SHANK éd., The Scientific Enterprise in Antiquity and the Middle Ages, Chicago, 2000, p. 215-231 ; G. SALIBA, « Seeking the origins of Modern Science», Bulletin of the Royal Institute for Inter-Faith Studies, 1/2 (1999), p. 139-152. G. SALIBA a écrit un livre dans lequel il traite de divers aspects d'importance historiographique, dont le déclin : Islamic Sciences and the Making of the European Renaissance, Cambridge (Mass.)/Londres, 2007 : mais ce livre est si trompeur et les affirmations si souvent superficielles, non étayées par des preuves ou simplement inventées, que je ne veux pas accabler le lecteur en lui recommandant de le lire.

11. Les principales conditions contextuelles des sciences mathématiques dans les sociétés islamiques sont assurées par leurs affiliations disciplinaires à travers les règles de succession, l'astrologie, la science du temps, la médecine, la géomancie, la magie des lettres, l'architecture, le commerce et l'arpentage, ainsi qu'à travers leurs espaces socioculturels dans les cours, les institutions d'enseignement, les administrations (surtout bureaux financiers), les armées et les autres officines chargées du contrôle des balances, ainsi qu'à travers les professions qui sont en relation avec les instruments et la construction ou l'organisation des installations hydrauliques.

12. D'autres présentations et appréciations de ces débats, avec lesquels je ne suis pas d'accord, se trouvent dans A. DaLlaL, Islam, Science, and the Challenge of History, New Haven/Londres, 2010, et J. Lyons, Islam Through Western Eyes : From the Crusades to the War on Terrorism, New York, 2012, p. 73-111. En particulier, les jugements de Lyons sont profondément erronés et témoignent plus d'une fois de son manque de compréhension du contenu et des contextes des sciences en pays d'Islam. Bien que les historiens des sciences en pays d'Islam aient eu sans aucun doute de nombreux préjugés qui ont façonné leurs intérêts et les résultats de leurs recherches, ils n'étaient pas aussi partiaux et primitifs que Lyons 
Lors des débats historiographiques sur le constructivisme social et ce qu'on appela les tournants linguistique, culturel, spatial, etc., les historiens des mathématiques et des sciences exactes en pays d'Islam sont restés pour la plupart silencieux, continuant à adhérer principalement à leurs programmes de recherche internalistes bien établis. Certaines modifications se sont toutefois produites, en ce qui concerne en particulier l'histoire de l'éducation et une mise en contexte plus étroite des textes mathématiques au sein des sphères intellectuelles de la philosophie, le kalām (la discussion rationnelle des questions de la foi) et le fiqh (le droit musulman). Dans des pays extérieurs au monde occidental, comme l'Iran, l'Algérie, le Maroc, la Tunisie, la Syrie, la Turquie ou la Chine, les histoires nationales des mathématiques et des sciences exactes, que ce soit dans un cadre laïque ou religieux, ont commencé à accorder une certaine attention aux institutions, aux méthodes et aux manuels scolaires écrits dans leurs langues et sur leurs propres territoires ${ }^{13}$. Parallèlement à ces changements, qu'on rencontre aussi bien chez les jeunes chercheurs que chez les chercheurs confirmés, les doctorants et les postdoctorants travaillant sur des dynasties et des périodes longues ont commencé à manifester davantage d'intérêt pour une étude localisée des questions mathématiques, astrologiques, divinatoires et d'autres sujets apparentés, prenant en compte leur relation avec la politique et les cultures courtisanes. Aujourd'hui, par conséquent, les jeunes chercheurs dans les différents domaines académiques entreprennent de manière plus sérieuse des recherches de type contextuel, y compris à propos des mathématiques et des autres matières d'enseignement.

\section{Que peut apporter l'étude de textes d'enseignement?}

De nombreux textes scolaires produits dans les madrasas, les mosquées ou des lieux privés sont élémentaires, répétitifs, simples, dépourvus de preuves et n'ayant nullement l'objectif de produire de nouveaux résultats

les décrit. Ce dernier a surtout utilisé des textes rédigés par des personnes ayant de fortes convictions nationalistes ou islamistes.

13. A. BAKI, Matematik Tarihi ve Felsefesi, Ankara, 2014 ; M. T. ToneKABOnI, « Kotob-e darsi-e qadim », Farhang-e Iran Zamin, 20 (1974), p. 39-60 ; D. LAMrABET, « Notes diverses sur l'enseignement des mathématiques au Maroc sous les Almohades (542-668 H/1147-1269) et les Mérinides (668-870H/1269-1465) », dans B. El BouazzATI éd., Les Institutions de sciences et d'enseignement au sein de la civilisation islamique, Rabat, 2008 ; M. ABDELJAOUAD, " History of Mathematics Education in the Islamic Countries in the Middle Ages », The International Journal for the History of Mathematics Education, vol. 7/1 (2012), p. 1-15 ; M. Abdeljaouad, « Chapter 20. L'enseignement des mathématiques dans les pays islamiques à l'époque moderne : Case Study of Tunisia », dans A. KARP et G. Schubring éd., Handbook on the History of Mathematics Education, Heidelberg, 2014, p. 405-428; Guo S. 郭书春 éd., Zhongguo Kexue Jishushi-Shuxue 中国科学技术史-数学 [The History of Science and Technology in China : Mathematics], Pékin, 2010. 
mathématiques ${ }^{14}$. Ces qualités sont dues au fait qu'ils étaient destinés avant tout à transmettre aux étudiants des connaissances et des compétences rudimentaires dans un certain nombre de disciplines mathématiques, en particulier l'arithmétique, l'algèbre et la connaissance du ciel, dans la mesure où ces disciplines étaient nécessaires pour ceux qui exerçaient une profession juridique ou astrologique, ou parce qu'elles faisaient partie du bagage intellectuel de tout érudit musulman bien formé et des membres des élites urbaines. À d'autres époques et dans d'autres régions, l'éventail des sujets enseignés différait de celui présenté ici parce que les conditions socioculturelles concrètes des activités intellectuelles étaient différentes ${ }^{15}$. Plusieurs régions de l'Est n'ont pas participé au développement de la nouvelle discipline relative à la mesure du temps, tandis que d'autres n'ont pas vu beaucoup de savants s'intéresser à la théorie planétaire. De telles différences entre le contenu thématique et technique des textes scolaires, entre leurs niveaux de difficulté et leurs méthodes de reproduction et de diffusion des connaissances mathématiques, nous donnent l'occasion d'aborder des questions historiographiques fondamentales.

L'une d'elle est de savoir si, en tant qu'historiens, nous devrions appeler les sciences que nous étudions les sciences arabes ou islamiques, les sciences en Islam ou plutôt les sciences au sein de sociétés islamiques (spécifiques).

Une deuxième question fondamentale est celle du Sitz im Leben des sciences mathématiques dans toute société islamique concrète, dans une ville, dans une ville de province, dans un village ou dans une forteresse, pour ne citer que les lieux les plus souvent mentionnés (lorsqu'ils le sont) dans les colophons de textes scientifiques arabes ou persans. Les textes scolaires, avec leurs notes marginales, les références, les feuilles volantes et les mentions relatives à la propriété, peuvent nous dire où les connaissances mathématiques étaient disponibles, qui a acquis les textes mathématiques et les écrits qui leur sont apparentés, ou encore quel était le lien entre les différentes disciplines et les textes enseignés dans les madrasas ou les mosquées à des moments ou en des emplacements spécifiques. L'étude des paratextes de tout texte mathématique, y compris de textes scolaires, devrait faire partie intégrante de notre analyse historique, comme je l'expliquerai dans la quatrième section de cette étude. L'étude des paratextes pour euxmêmes, c'est-à-dire quasi indépendamment du texte dans lequel ils sont inscrits, est une autre option pour acquérir une meilleure compréhension des approches pédagogiques pratiquées dans des circonstances concrètes ou sur la longue durée.

14. Voir, par exemple, S. P. RaGEP, Jaghmīnī's Mulakhkhaș. An Islamic Introduction to Ptolemaic Astronomy, Heidelberg, 2016.

15. Voir S. BRENTJES, Teaching and Learning the Sciences in Islamicate Societies... 
Une troisième question fondamentale est celle de savoir ce qu'il est possible de faire pour mieux comprendre les conditions dans lesquelles les textes mathématiques et les textes scolaires connexes ont été produits, utilisés, diffusés et valorisés, au-delà de l'information qu'on trouve dans ces textes eux-mêmes et dans leurs contenants matériels, à savoir les manuscrits. $\mathrm{J}$ 'aborde cette question dans la cinquième et dernière section de cette étude et propose d'analyser surtout les dictionnaires biographiques comme une source complémentaire des plus précieuses, en plus des textes mathématiques proprement dits. Mais d'autres types de sources devraient aussi être pris en considération, notamment les carnets de notes, les dossiers de cours, les registres fiscaux et les calculs isolés, insérés sur les pages de couverture ou ailleurs dans des manuscrits et dépourvus de tout autre contexte.

\section{Les débats dichotomiques sur l'éducation dans les madrasas et les mosquées versus l'enseignement dans les maisons privées}

En 1981, George Makdisi déclara catégoriquement que les sciences anciennes n'étaient pas enseignées dans les madrasas car il s'agissait d'institutions religieuses destinées à enseigner les doctrines des différentes écoles du droit musulman. Il reconnaissait cependant que cela ne signifiait pas que les sciences anciennes n'étaient pas du tout enseignées et qu'elles avaient progressivement disparues. Il ne manquait pas d'insister ${ }^{16}$ :

L'autre division, appelée « les sciences des Anciens », c'est-à-dire celle des Grecs, bien que contestée pour ses principes « païens » par tout savant musulman croyant parmi les fidèles, exigeait néanmoins un respect non exprimé publiquement, silencieux et réservé. Ces sciences étaient enseignées en privé et étaient exclues des cours réguliers des institutions musulmanes d'enseignement.

Bien sûr, des témoignages dans les manuscrits confirment parfois de telles séances d'enseignement privées. Mais d'autres témoignages ne laissent aucun doute sur le fait que les sciences mathématiques ont été enseignées dans les madrasas, les mosquées et les loges soufies ${ }^{17}$.

Malgré tout, le livre substantiel et largement diffusé de G. Makdisi sur les madrasas, leur structure juridique et leurs pratiques d'enseignement, a permis comme aucun autre de comprendre la place des mathématiques et des sciences apparentées dans le milieu éducatif des sociétés islamiques

16. "The other division, called "the sciences of the Ancients", that is of the Greeks, while opposed for its "pagan" principles by every believing Muslim scholar among the faithful, commanded nevertheless an unpublicized, silent, begrudging respect. These sciences were taught in private, and were excluded from the regular courses of Muslim institutions of learning » (G. MAKDISI, The Rise of Colleges..., p. 75-76).

17. S. BRENTJES, Teaching and Learning the Sciences in Islamicate Societies..., p. 77-111. 
pendant plusieurs décennies. En étudiant la relation entre la thèse de la marginalité et la thèse selon laquelle les madrasas (ou les mosquées) avaient fermé leurs portes aux sciences anciennes, A. I. Sabra a eu raison de soutenir qu'on ne saurait se contenter de rendre ce fait (présumé) responsable du déclin des sciences que l'on avait diagnostiqué, mais que nous devons aussi expliquer les facteurs qui ont produit ce fait (présumé) et chercher à savoir comment « une tradition scientifique significative s'est maintenue pendant si longtemps, en grande partie en dehors de la seule institution d'enseignement supérieur stable de l'Islam médiéval ${ }^{18} »$. Il a considéré la thèse du déclin (présumé) avec une certaine hésitation, en reconnaissant « que l'activité scientifique n'a pas cessé d'exister parce qu'elle n'était pas formellement incluse dans le programme d'enseignement de la madrasa », soulignant en même temps « que, grâce au caractère informel de toute éducation musulmane médiévale, les sciences rationnelles, ou certaines d'entre elles, ont même pu pénétrer les madrasas ${ }^{19}{ } »$.

L'idée que l'éducation à la madrasa et à la mosquée avait un caractère informel a été renforcée par les études de Jonathan Berkey et Michael Chamberlain sur les disciplines religieuses ${ }^{20}$. Elle est fondée sur l'accent mis par les sources historiques sur les personnes et les textes et non sur les institutions, ce qui signifie qu'aucun programme formel n'existait avant 1500. Les donateurs, les enseignants et les étudiants étaient libres de déterminer la progression, la durée et l'organisation thématique de leur enseignement ou de leurs études. Il n'existait pas de système d'examens prescrits de manière régulière et de règles officielles pour évaluer les connaissances et les compétences acquises. La seule façon de confirmer la participation d'un élève à des activités de nature éducative était l'autorisation écrite donnée par un enseignant (ijāza) de lui dispenser un enseignement spécifique sur le texte étudié avec lui à travers l'une des principales formes reconnues dans ce système éducatif informel, à savoir la lecture d'un texte avec un enseignant, le fait d'écouter parler un enseignant ou encore d'étudier en la présence d'un enseignant. Ces autorisations d'enseigner

18. A. I. SABRA, «The Appropriation and Subsequent Naturalization of Greek Science in Medieval Islam »..., p. 16.

19. Ibid., p. 16. L'expression sciences rationnelles est souvent mal comprise par les historiens qui la considèrent comme identique à sciences anciennes. Alors que la philosophie, la médecine, les sciences mathématiques et les disciplines occultes faisaient partie de ces dernières, la philosophie et la logique ont été associées aux disciplines religieuses fondées sur des arguments et des démonstrations rationnels comme l'étaient les disciplines philologiques. Les sciences mathématiques se trouvent souvent dans leur propre section au sein des classifications des sciences, bien que dans les dictionnaires biographiques les frontières entre elles et les sciences rationnelles soient souvent floues (S. BRENTJES, Teaching and Learning the Sciences in Islamicate Societies..., p. 187-221).

20. J. P. Berkey, The Transmission of Knowledge in Medieval Cairo. A Social History of Islamic Education, Princeton (NJ), 1992 ; M. ChamberLAIN, Knowledge and Social Practice in Medieval Damascus, 1190-1350, Cambridge, 1995. 
étaient principalement accordées dans les disciplines religieuses. Certaines sont aussi connues pour des traités de médecine. Mais elles n'existent que de manière exceptionnelle pour les textes mathématiques, même si les plus anciens certificats d'enseignement existants que je connaisse proviennent, sous la forme de déclarations sur la dictée et l'écoute, de textes mathématiques du IX $\mathrm{X}^{\mathrm{e}}$ siècle $^{21}$. Étant donné qu'aucun effort n'a été fait jusqu'à présent pour recueillir de telles permissions dans le domaine des sciences mathématiques, on ne sait pas exactement ce que signifie leur absence pour les textes mathématiques. Cependant, d'après l'étude de Stefan Leder, alSawās et al-Sāgharjī sur les certificats d'audition en sciences religieuses, nous savons que la délivrance de tels documents ne dépendait pas des lieux d'enseignement et d'apprentissage (madrasa, mosquée, maison privée, tombeau, loge, jardin $)^{22}$. L'absence de telles permissions dans la plupart des copies existantes de textes mathématiques ne peut donc pas être interprétée comme un signe que ces textes n'étaient pas enseignés dans les madrasas ou les mosquées.

L'accent mis sur les personnes dans les pratiques qui valorisent l'éducation va cependant de pair avec une plus grande organisation institutionnelle des activités pédagogiques. Les institutions étaient dotées du cadre juridique des donations religieuses (waqf), qui comprenait des dispositions sur le personnel, les salaires et les rémunérations, le financement et l'administration, ainsi que les dispositions matérielles. Un autre élément qui contribua à rendre l'enseignement plus formel fut l'émergence de corpus canonisés de textes d'enseignement, de méthodes communes d'enseignement et d'apprentissage et de genres littéraires spécifiques permettant aux gens de rapporter et d'enregistrer leurs expériences d'apprentissage (dictionnaires d'enseignants et livres, récits de voyages mettant l'accent sur l'éducation, dictionnaires de savants) ${ }^{23}$. Nous ne comprenons pas bien comment ce processus de canonisation des sources, des valeurs et des pratiques a pu voir le jour et fonctionner. Après tout, il n'existait aucune structure institutionnelle au sein de laquelle ces questions ont pu être réglées. Il est clair qu'elles différaient d'une région du vaste monde des pays d'Islam à l'autre, comme au fil du temps. L'absence d'études systématiques du matériel et des méthodes d'enseignement des grands centres et des villes de province - ne serait-ce que pour une seule période - ne permet pas de

21. S. BRentJes et J. RenN, « Contexts and Content of Thābit ibn Qurra's (died 288/901) Construction of Knowledge on the Balance », dans S. BRENTJES et J. RENN éd., Globalization of Knowledge in the Post-Antique Mediterranean. 700-1500, Londres/New York, 2016, p. 67-100.

22. S. Leder, Y. M. AL-SAWĀs, M. AL-SĀGHARJī, Mu 'jam al-samā 'āt al-dimashqīya almuntakhaba min sanati 550 ilā 750 . Les certificats d'audition à Damas, 550-750 h./1155 à 1349, Damas, 1996.

23. S. BrentJes, Teaching and Learning the Sciences in Islamicate Societies..., p. 147186 et $223-262$. 
formuler d'hypothèses qui soient suffisamment fondées. J'ai toutefois plaidé pour que l'on considère les dictionnaires biographiques comme des outils destinés à contrôler et à orienter les communautés de savants. Puisqu'ils n'établissent pas de différence d'appréciation sensible entre, d'une part, l'éducation acquise dans les madrasas et les mosquées, et d'autre part, celle dispensée dans les maisons privées, j'ai suggéré d'accepter la perspective propre à ces outils au lieu de nous en tenir à une vision moderne qui tend clairement à privilégier l'éducation au sein d'une institution ${ }^{24}$. Je pense qu'il n'est pas seulement anhistorique, mais aussi peu judicieux de batailler pour savoir si les disciplines mathématiques, philosophiques, médicales et occultes étaient enseignées dans les madrasas, les mosquées ou les loges soufies, ou uniquement dans les maisons privées des enseignants ou des étudiants. Les étudiants comme les enseignants vivaient souvent dans des madrasas ou des loges soufies, en particulier lorsqu'ils venaient d'autres grandes villes, de petites villes de province ou de villages. Cette position a toutefois été récemment rejetée par Sally P. Ragep comme diminuant de manière indue le rôle de la structure institutionnalisée de l'enseignement et de l'apprentissage ${ }^{25}$.

\section{Sources d'information complémentaires sur l'enseignement des mathématiques (paratextes)}

Les sources d'information sur les lieux d'enseignement et d'apprentissage qui complètent les traités mathématiques sont les éléments paratextuels qui se trouvent dans les marges, au début ou à la fin des traités mathématiques, sur des feuilles volantes ainsi que sur les pages de couverture et les frontispices. Dans le cas de codices à textes multiples et de codices où sont reliés plusieurs traités, les paratextes peuvent également se trouver sur des pages qui, avant d'être insérées entre des textes différents, étaient à l'origine vides. De tels paratextes offrent un large éventail d'informations. Ils nous révèlent les noms et, parfois, l'identité professionnelle des scribes, de lecteurs, de vendeurs et d'acheteurs de ces textes ou codices, les institutions auxquelles les manuscrits étaient destinés ou dans lesquelles ils étaient conservés, et donc leur circulation. Ils nous disent si un scribe a copié un texte pour son propre usage ou pour un client, la date de la copie, de la correction et le coût des manuscrits. Les notes marginales nous informent

24. EAD., « On the Location of the Ancient or "Rational" Sciences in Muslim Educational Landscapes... », p. 60-61.

25. S. P. RaGeP, « Fifteenth Century Astronomy in the Islamic World », dans R. FeldhaY et F. J. Ragep éd., Before Copernicus : The Cultures and Contexts of Scientific Learning in the Fifteenth Century, Montréal, 2017, p. 143-160, surtout p. 155. Malheureusement, Sally P. Ragep déforme ma position et montre qu'elle comprend mal la nature du problème. 
le plus souvent sur le nombre d'utilisateurs et les contextes dans lesquels ils ont étudié un texte ou un codex tout entier, sur le type de questions qu'ils ont posées, sur les exercices qu'ils ont effectués, sur les autres textes qu'ils ont consultés en étudiant un texte donné et sur quelques-unes des méthodes utilisées par les enseignants.

Une étude systématique des données géographiques, institutionnelles, économiques ou prosopographiques améliorerait considérablement notre connaissance de l'enseignement des mathématiques, de la diffusion des textes et des manuscrits, ainsi que de l'entourage et des statuts sociaux des lecteurs. C'est surtout à partir des codices et de leurs textes mathématiques qu'on peut se faire une idée du rôle ordinaire des connaissances mathématiques dans la vie quotidienne de leurs propriétaires et utilisateurs.

Les choses ne sont cependant pas toujours simples et explicites. Par exemple, la majorité des textes scolaires existants ne contiennent aucune information sur l'endroit où ils ont été écrits, lus ou commentés. Seule une minorité de textes possède des colophons qui précisent, en plus du scribe et de l'année de la copie, la localité et l'emplacement précis où travaillait le scribe. Les endroits mentionnés varient entre de grandes villes, de petites villes de province, des villages et des résidences royales d'été. Les cadres spécifiques des lieux de production des copies incluent des forteresses, des loges religieuses, des madrasas et des maisons privées.

Plusieurs problèmes méthodologiques doivent être abordés afin de pouvoir interpréter de manière significative et fiable ces données et leur relative rareté. Gerhard Endreß aborde par exemple l'un de ces problèmes lorsqu'il est amené à douter que le fait de copier un texte dans une madrasa signifie nécessairement qu'il y est aussi enseigné et étudié ${ }^{26}$. À première vue, le doute de G. Endreß semble injustifié car nous savons que la copie de textes était une pratique majeure de l'enseignement et de l'apprentissage, condition préalable à l'obtention d'une permission d'enseigner (ijazza) et à la possession du texte étudié. Il n'est pas inapproprié pour autant d'adopter une position prudente car la copie de textes était aussi la principale activité pour la gestion financière d'une bibliothèque, pour gagner sa vie ou faire un don. Afin d'acquérir une connaissance plus assurée de ce que pourrait signifier l'attribution de textes copiés à des lieux spécifiques et de ce que pourraient signifier - d'un point de vue sociologique de manière générale et plus spécifiquement d'un point de vue institutionnel - les copies de textes mathématiques qui sont le plus souvent non attribués, non datés et non signés, nous devons donc, d'une part, explorer d'autres matériaux que les seules copies de textes mathématiques, et d'autre part, poser des questions

26. G. ENDRESS, «Reading Avicenna in the Madrasa : Intellectual Genealogies and Chains of Transmission of Philosophy and the Sciences in the Islamic East », dans J. E. Montgomery éd., Arabic Theology, Arabic Philosophy : From the Many to the One, Louvain, 2006, p. 371-422. 
sur les textes mathématiques préservés qui dépassent leur contenu et les aspects relativement simples comme les endroits où ils ont été produits, lus ou enseignés. Il nous faut dès lors nous renseigner sur les hiérarchies de pertinence des textes et des enseignants, la richesse et le statut social des lieux d'enseignement et de copie, les réseaux auxquels appartenaient les enseignants de mathématiques, les alliances et les amitiés qui les reliaient à d'autres enseignants et aux fonctionnaires des cours en ville, et les façons dont certains élèves ont acquis par la suite une importance intellectuelle et socioculturelle, tandis que d'autres n'apparaissent pas dans les récits consacrés à l'enseignement et à l'apprentissage.

\section{Matériel supplémentaire pour une histoire de l'enseignement des mathématiques}

Comme je l'ai indiqué précédemment, les dictionnaires biographiques me paraissent constituer le complément le plus important pour étudier l'enseignement des mathématiques. Les chroniques historiques forment une autre source, mais elles apportent souvent moins d'informations. D'autres sources pour élaborer une histoire de l'enseignement des mathématiques au sein de sociétés islamiques particulières sont les carnets de notes, les listes d'enseignants et les livres étudiés et/ou compilés par d'anciens élèves, les récits de voyage des élèves ou des enseignants et les catalogues de bibliothèques. L'étude de la composition de codices entiers apporte également un ensemble riche d'informations utiles sur la distribution des textes mathématiques dans ces collections et donc sur les conceptions des bibliothécaires, des collectionneurs de livres, des vendeurs de livres ou des personnes copiant et lisant des textes pour un usage privé. Dans certains cas, les codices composites et les codices à textes multiples comprennent des informations sur leur(s) vie(s) antérieure(s), avant que les codices actuels n'aient été confectionnés ou reliés ensemble à partir de textes d'origines, d'âges et d'objectifs différents. Ces indications rendent compte de l'origine géographique de ces textes (qu'il s'agisse d'une simple partie ou de la totalité), de la période où ils furent utilisés, ou encore de la circulation de chaque partie, de l'ensemble d'un recueil ou de collections appartenant à un même groupe d'utilisateurs.

La présence de ce type de matériel alternatif dans les sociétés islamiques et au cours des siècles varie cependant considérablement d'un pays à l'autre. Alors que, par exemple, les chroniques historiques ont été écrites pour de nombreuses dynasties et localités, les dictionnaires biographiques des savants sont moins fréquents. Les historiens de nombreuses régions, localités ou dynasties n'estimaient pas qu'il fût de leur devoir d'immortaliser les savants par l'intermédiaire d'une biographie. C'est pourquoi les spécialistes des disciplines mathématiques, philosophiques, 
médicales et occultes n'ont pas souvent fait l'objet de notices biographiques. En outre, très peu de dictionnaires exclusivement dédiés à leurs domaines de connaissance furent réalisés, alors qu'on produisit en bien plus grande quantité des dictionnaires biographiques consacrés à des savants dont la discipline appartient à la religion (comme le droit qu'impliquent les principales doctrines juridiques sunnites ou chiites), à la transmission des hadìth ou aux lecteurs et aux exégètes du Qur'an.

Des listes d'enseignants et de livres étudiés sont surtout connues - du moins par moi - pour al-Andalus, l'Afrique du Nord et l'Égypte, à partir $\mathrm{du}$ XIII $^{\mathrm{e}}$ siècle environ. Quelques-unes d'entre elles seulement donnent des informations sur les sciences mathématiques, bien qu'aucune exploration systématique de ce type de matériel n'ait été menée à ce jour. En revanche, des carnets de note semblent avoir été principalement compilés dans des régions plus à l'est, en Iran, en Anatolie ou en Inde. Plusieurs de ceux que j'ai vus dans les bibliothèques de manuscrits contiennent des citations de textes mathématiques ou des discussions relatives à des problèmes mathématiques. Quelques-uns se composent même presque exclusivement de matériel mathématique. Comme ils sont souvent anonymes et non datés, il est très difficile de les situer avec certitude dans le cadre d'un enseignement au sein des madrasas et des mosquées. Les caractéristiques structurelles de l'enseignement dans les madrasas, énumérées ci-dessus, suggèrent plutôt que ces carnets devaient être le résultat de pratiques de lecture privées et d'emprunts à la littérature savante. La plupart des catalogues de bibliothèques que je connais provient de périodes postérieures au $\mathrm{Xv}^{\mathrm{e}}$ siècle et, principalement, des cours princières, même si quelques listes de livres de ce genre ont été publiées et discutées par Konrad Hirschler et Doris Behrens-Abouseif, à propos d'un souverain ayyoubide (XIII ${ }^{\mathrm{e}}$ siècle) et d'un médecin mamelouk $\left(\mathrm{XV}^{\mathrm{e}} \text { siècle }\right)^{27}$.

Alors qu'il est possible de trouver, y compris dans les dictionnaires spécialisés consacrés à ceux qui ont transmis les hadīth ou aux lecteurs et exégètes du Qur'an, des informations sur telle ou telle personne impliquée dans les sciences mathématiques, ces types de dictionnaires présentent dans l'ensemble une image qui confirme à la fois la thèse antiscientifique d'Ignaz Goldziher et la thèse de marginalisation des madrasas de G. Makdisi ${ }^{28}$. En revanche, les dictionnaires biographiques consacrés aux savants exerçant dans le domaine du droit et aux juristes nous permettent de dire qu'une

27. K. HirSChLER, Medieval Damascus : Plurality and Diversity in an Arabic Library. The Ashrafiya Library Catalogue, Edinburgh, 2016 ; D. Behrens-ABouseIF, « The Waqf of a Cairene Notable in early Ottoman Cairo-Muhibb al-Din Abu al-Tayyib, fils d'un médecin », dans R. Deguilhem éd., Le Waqf dans l'Espace Islamique-Outil de pouvoir socio-politique, Damas, 1995, p. 123-132.

28. I. GoldZIHER, "The Attitude of the Old Islamic Orthodoxy Toward the "Ancient Sciences" »..., p. 185-215 ; G. MAKdISI, The Rise of Colleges... 
telle vision n'est pas toute la vérité. Ils contiennent en effet souvent des informations sur les auteurs de traités mathématiques, philosophiques ou médicaux en prose et sur la poésie didactique.

Les dictionnaires des personnages importants sur un siècle donné et les dictionnaires des fils importants de tous les temps confirment que beaucoup plus d'élèves qu'on ne pensait ont suivi au moins quelques cours dans les sciences mathématiques, en particulier en arithmétique et sur le calcul du temps, mais souvent aussi en algèbre, en géométrie et en théorie planétaire élémentaire. Ce type de dictionnaire peut inclure des savants préislamiques, surtout des philosophes, des médecins ou des mathématiciens grecs anciens, et donne également une place importante aux savants chrétiens et juifs appartenant aux disciplines mathématiques, à la philosophie et à la médecine. La première catégorie comprend les savants de sexe masculin, et à l'occasion des femmes enseignant le droit et les hadīth, en compagnie d'administrateurs financiers et diplomatiques, de dirigeants et de militaires de rang supérieur. Avec les rares dictionnaires biographiques principalement dédiés aux médecins ou aux philosophes (qui comptaient dans leurs rangs ceux qui se consacraient surtout aux sciences mathématiques), les dictionnaires biographiques des savants en droit et des juristes, ainsi que les deux types de dictionnaires plus généraux cités précédemment, nous fournissent un large éventail d'informations sur les personnes qui ont enseigné,étudié ou exercé une ou plusieurs des disciplines mathématiques. Une exploration systématique de ces dictionnaires pourrait par conséquent nous permettre d'établir des descriptions plus complètes et en même temps plus nuancées de ceux qui, parmi les savants d'une ville ou d'un village, se sont consacrés aux mathématiques (quand c'est le cas), sous quelles formes et avec quelles disciplines particulières. Malheureusement, aucune enquête systématique n'existe encore pour quelque localité ou période que ce soit.

Les études actuellement disponibles, de nature plus limitée, confirment néanmoins que, pour des localités et des périodes spécifiques, aucune des trois principales thèses historiographiques du passé (le déclin, la marginalisation ou l'opposition générale antiscientifique) ne se vérifie. S'appuyant sur des textes mathématiques et astronomiques ainsi que sur des instruments, David A. King et François Charette ont montré que les muwaqqitūn travaillant dans les madrasas ou les mosquées ayyoubides et mameloukes du Caire et de Damas inventèrent de nouveaux instruments, conçurent des fonctions auxiliaires nécessaires pour le calcul de tables des heures de prière et pour la résolution de divers autres problèmes astronomiques avec plusieurs milliers d'entrées multiples. Ils réorganisèrent aussi certaines parties de la géométrie sphérique et de l'astronomie afin que la nouvelle discipline appelée «science du temps » puisse prospérer comme une véritable activité intellectuelle, qu'elle soit enseignée et 
puisse acquérir ainsi une reconnaissance sociale et culturelle ${ }^{29}$. David A. King, Daniel Varisco et Petra Schmidl ont édité, traduit et analysé des travaux astronomiques et astrologiques des membres de la dynastie des Rasulides des XIII ${ }^{\mathrm{e}}$ et $\mathrm{XIV}^{\mathrm{e}}$ siècles, démontrant que cette famille dirigeante et les savants qui lui étaient rattachés étaient bien informés dans ces deux disciplines et qu'ils avaient donc reçu une solide éducation en astronomie et en astrologie ${ }^{30}$. De nouveaux résultats mathématiques et des activités régulières d'enseignement sont également attestés pour plusieurs villes d'Afrique du Nord, dont Fès, Tlemcen et Tunis, grâce aux recherches menées par Ahmed Djebbar, Driss Lamrabet, Mahdi Abdeljaouad, Jeffrey Oaks et d'autres collègues ${ }^{31}$.

Mon analyse de l'un des principaux dictionnaires biographiques, compilé au $\mathrm{XV}^{\mathrm{e}}$ siècle par Shams al-Dīn al-Sakhāwī (1428-1497), un spécialiste en hadīth, confirme ces études fondées sur le contenu des textes mathématiques et astronomiques ${ }^{32}$. Elle présente l'image d'une communauté mathématique très vivante dans les madrasas, les loges soufies et quelques mosquées du Caire, dans lesquelles plusieurs enseignants se sont spécialisés dans différentes disciplines mathématiques. Les élèves pouvaient les choisir en fonction de leur personnalité et de leurs spécialités. Je considère cette communauté comme une « communauté vivante » parce qu'un groupe stable et diversifié d'enseignants et d'élèves semble s'être formé, qu'il s'est perpétué tout au long $\mathrm{du} X \mathrm{Xv}^{\mathrm{e}}$ siècle et qu'il a réussi à attirer dans le cadre de cette éducation des élèves qui s'intéressaient principalement aux sciences religieuses et qui n'utilisèrent pas, dans la suite de leur existence, la formation qu'ils avaient acquise afin de rédiger des textes mathématiques. Ce caractère vivant se reflète également dans la quantité et la diversité des textes écrits par ceux qui occupèrent des charges d'enseignants dans les madrasas, les mosquées ou les loges soufies, qui

29. D. A. KING, In Synchrony with the Heavens : Studies in Astronomical Timekeeping and Instrumentation in Medieval Islamic Civilization, vol. 1 : The Call of the Muezzin (Studies I-IX), vol. 2 : Instruments of Mass Calculation (Studies X-XVIII), Leyde, 2004, 2005 ; F. Charette, Mathematical Instrumentation in 14th-Century Egypt and Syria : The Illustrated Treatise of Najm al-Dīn al-Mișrī, Leyde, 2003.

30. D. A. KIng, Mathematical Astronomy in Medieval Yemen, Malibu (CA), 1983 ; D. M. V ARISCO, Medieval Agriculture and Islamic Science : The Almanac of a Yemeni Sultan, Seattle, 1994 ; P. SCHміDL, Volkstümliche Astronomie im islamischen Mittelalter. Zur Bestimmung der Gebetszeiten und der Qibla bei al-Aṣbah̄ì, Ibn Rah̄i q und al-Fārisī, Leyde, 2007.

31. A. DuebBar, Mathématiques et mathématiciens dans le Maghreb médiéval (IXe-XVI $s$.). Contributions à l'étude des activités scientifiques de l'Occident Musulman, HDR, Université de Nantes, 1994 ; D. LAMraBET, Introduction à l'histoire des mathématiques maghrébines, Rabat, 1994 ; M. ABdelJAOUAD et J. OAKs, « Al-Hawārî’s commentary on Ibn al-Bannā"s Talkhīṣ Contents and influences », Suhayl, 12 (2013), p. 9-44.

32. S. BRENTJES, "Euclid's Elements, courtly patronage and princely education »..., p. 323-341 ; EAD., Teaching and Learning the Sciences in Islamicate Societies..., p. 77-111, $147-185$ et $223-265$. 
dispensèrent des cours aux orphelins, qui construisirent et entretinrent des instruments publics et privés, ou qui furent engagés comme muwaqqitūn dans les mosquées et les madrasas.

Beaucoup, parmi les quelques 10000 personnes inscrites dans le livre d'al-Sakhāwī, n'ont pas suivi de cours en sciences mathématiques, mais certains l'ont fait. Afin d'obtenir une estimation quantitative, j'ai compté les étudiants et leurs orientations disciplinaires sur environ $20 \%$ des entrées (soit 1948 personnes). La longueur des entrées varie entre une demi-ligne et plusieurs pages. L'information fournie est donc inégalement répartie. Al-Sakhāwī mentionne 112 activités d'étudiants dans toutes les disciplines mathématiques qu'il reconnait à l'aide d'une terminologie technique comme « arithmétique », « géométrie » ou « théorie planétaire ». Si l'on suppose que le taux de ces activités est demeuré relativement stable - ce qui, bien sûr, n'est pas garanti -, les élèves auraient suivi environ 600 leçons de mathématiques durant le siècle tout entier. Comment évaluer un tel chiffre ? D'après mon estimation, qui ne repose pas sur un calcul, ce chiffre représente au plus $5 \%$ de tous les cours enseignés pendant cette période, tels qu'ils sont renseignés par al-Sakhāwī. Cela ne veut pas dire que ce dernier a entendu parler de tous les cours que les étudiants ont suivis tout au long du siècle ou qu'il a rapporté tous les cours au sujet desquels il avait reçu des informations. Nous ne savons pas vraiment quels étaient les critères de sélection d'al-Sakhāwī, parce qu'il n'existe aucune étude analytique de son dictionnaire biographique (comme de tout autre dictionnaire) pour ce qui concerne les sciences mathématiques, philosophiques, médicales et occultes. Aussi est-il tout au plus possible de conclure, à partir des entrées de cette liste, que les élèves ont bien étudié des textes mathématiques au $\mathrm{Xv}^{\mathrm{e}}$ siècle et qu'ils avaient même certaines préférences (Tableau 1).

\begin{tabular}{|l|c|}
\hline \multicolumn{1}{|c|}{ Disciplines } & Nombre d'élèves \\
\hline arithmétique + calculs d'héritage & 56 \\
\hline science du temps & 11 \\
\hline astrologie & 4 \\
\hline géométrie & 8 \\
\hline théorie planétaire & 7 \\
\hline algèbre & 10 \\
\hline manuels d'astronomie & 5 \\
\hline éphémérides & 3 \\
\hline instruments & 3 \\
\hline arpentage & 5 \\
\hline
\end{tabular}

Tableau 1 : Nombre d'élèves par discipline mentionnés dans le dictionnaire biographique de Shams al-Dīn al-Sakhāwī. 
Al-Sakhāwī était lui-même l'un de ces élèves qui suivirent des cours de mathématiques. Il a étudié avec plusieurs enseignants, parmi lesquels le principal savant et enseignant de sciences mathématiques au Caire dans la première moitié du Xv $\mathrm{Xv}^{\mathrm{e}}$ siècle, Ibn al-Majdī (m. 1447). Al-Sakhāwī a beaucoup apprécié son professeur et lui a dédié une longue entrée ${ }^{33}$. Il n'était pas le seul habitant important de la ville à avoir une haute opinion d'Ibn al-Majdī. Les sultans mamelouks de la première moitié du XV siècle lui demandèrent conseil et l'invitèrent au palais. Selon les données d'alSakhāwī, Ibn al-Majdī comptait environ dix-huit élèves. Plusieurs d'entre eux sont devenus des enseignants de sciences mathématiques (y compris pour le calcul du temps). Pour François Charrette, la plupart des traités astronomiques d'Ibn al-Majdī sont didactiques, c'est-à-dire rédigés en vue d'être enseignés ${ }^{34}$. Cela s'applique probablement aussi à ses textes d'arithmétique, d'algèbre, de théorie des nombres, de géométrie et ses autres textes mathématiques qui n'ont pas encore été suffisamment étudiés.

Comme l'indique le Tableau 1, les élèves qui figurent dans les entrées d'al-Sakhāwī ont participé à différents types de cours. La plupart d'entre eux ont reçu une instruction dans une classe de droit spécialement consacrée à une série de procédures mathématiques destinées à déterminer les parts d'héritage pour chacun des membres d'une famille, ainsi que le calcul des dons et des autres types de donations qu'un propriétaire pouvait faire à des personnes étrangères à sa famille. J'ai exclu, dans le Tableau 1, ce domaine de la formation mathématique lorsqu'il apparaissait seul, parce que je me suis intéressée seulement aux disciplines qui composent l'une des branches des sciences mathématiques dans les traités médiévaux portant sur la classification des sciences. Le deuxième type de cours le plus fréquenté combinait ce domaine juridique avec l'enseignement de différents systèmes de numération et de calculs en chiffres indiens (que nous appelons aujourd'hui arabes), en chiffres alphanumériques, au moyen des phalanges des doigts de la main et avec d'autres types d'opérations réalisées oralement. Un petit nombre d'élèves leur ajoutait des cours sur l'algèbre et, parfois, sur la géométrie, ainsi que sur différents domaines de l'astronomie (théorie planétaire, lecture de manuels d'astronomie, science du temps, construction d'almanachs et d'instruments). Enfin, certains élèves n'ont choisi, comme sujets d'études en sciences mathématiques, que la théorie planétaire et la géométrie ${ }^{35}$.

Les enseignants du Caire complétèrent ce tableau au $\mathrm{Xv}^{\mathrm{e}}$ siècle en proposant différentes combinaisons disciplinaires pour leurs enseignements. Plus d'une vingtaine d'enseignants de sciences mathématiques sont cités

33. Shams al-Dĩn Al-SaKhāwī, Al-Ḍaw' al-lāmi li-ahl al-qarn al-tāsi ', Beyrouth, n.d. (10 vol.), vol. 1, p. 300-302.

34. F. ChARETTE, « Ibn al-Majdī », dans Th. Hockey et al. éd., Biographical Encyclopedia of Astronomers, New York, 2007 (2 vol.), vol. 1, p. 561-562.

35. S. BRENTJES, Teaching and Learning the Sciences in Islamicate Societies..., p. 163-164. 
dans le premier volume d'al-Sakhāwi ${ }^{36}$. Au moins les deux tiers d'entre eux ont enseigné la science du droit successoral en utilisant des méthodes mathématiques et l'arithmétique. Les autres enseignaient soit d'autres disciplines, soit davantage que ces deux types de cours. La plupart de ces noms sont inconnus des historiens des sciences mathématiques, soit parce que leurs traités n'ont pas encore été étudiés ou découverts, soit parce qu'ils n'ont laissé aucune trace de cette nature. Certains sont bien connus comme enseignants de sciences religieuses. Dès lors qu'ils nous sont présentés par l'une des entrées biographiques d'al-Sakhāwī et qu'ils ont même laissé quelques traités, ils pourraient également trouver leur place dans l'histoire des mathématiques. Même les grands professeurs de sciences mathématiques enseignaient très régulièrement le droit, les composantes religieuses des sciences rationnelles et une ou plusieurs des disciplines suivantes : philologie, rhétorique, logique et philosophie. Ibn al-Majdī et al-Kafiyajī (m. 1474) faisaient partie de ceux qui n'enseignaient pas uniquement les mathématiques liées aux héritages et l'arithmétique. Ibn al-Majdi a donné des cours sur des textes appartenant à presque tous les domaines mathématiques disponibles, en particulier au calcul du temps, à l'arithmétique, à l'algèbre et à la géométrie. Il a également enseigné le droit, la langue arabe et la philosophie. Son élève Ali, honoré par l'épithète Tilmīdh Ibn al-Majdī (élève d'Ibn al-Majdī), a enseigné l'arithmétique, l'algèbre et le calcul du temps ${ }^{37}$. Al-Kafiyajī était quant à lui un éminent professeur de sciences rationnelles. Il a enseigné en outre la géométrie et la théorie planétaire ${ }^{38}$.

À l'aide du même échantillon, j'ai également compté le nombre d'enseignants en fonction des disciplines dont ils avaient la charge (Tableau 2). Ils ne correspondent pas toujours aux entrées des élèves, car al-Sakhāwī ne nomme pas toujours les enseignants qu'ils ont pu avoir dans chaque domaine d'études. À l'inverse de cet écart dû à la méthode suivie par al-Sakhāwī, nous pouvons supposer - avec plus ou moins de certitude - que non seulement les activités liées à l'enseignement correspondaient à l'investissement des élèves dans les sciences mathématiques (puisque les textes pédagogiques produits au $\mathrm{Xv}^{\mathrm{e}}$ siècle dans les localités énumérées par al-Sakhāwī supposent leur inclusion dans des enseignements réguliers), mais aussi que, selon toute vraisemblance, le nombre de ces activités a atteint un chiffre plus élevé que celui donné par al-Sakhāwī. Cela est suggéré par deux de ses exemples. Dans le premier, al-Sakhāwī cite une activité d'enseignement d'Ibn al-Majdī en relation avec Sibṭ al-Māridānī (14231506), l'un de ses élèves qui fut lui-même, par la suite, un enseignant très prolifique et très lu. Il précise que les deux hommes ont étudié de nombreux

36. Shams AL-Dĩn AL-SAKHĀwī, Al-Daw' al-lāmi 'li-ahl al-qarn al-tāsi '..., vol. 1, p. 22, 25-26, 49, 60, 82, 86, 100-101, 124, 126, 128, 134, 204-205, 218, 225, 227-230, etc.

37. Ibid., vol. 1, p. 100-101.

38. Ibid., vol. 7, p. 259-260. 
textes écrits par Ibn al-Majdī ${ }^{39}$. Une entrée pour un enseignant représente donc, ici, plusieurs cours différents. Dans l'autre exemple, al-Sakhāwī raconte à ses lecteurs que quelqu'un a présenté un élève à ses professeurs de religion et qu'il a pris ensuite avec eux des cours de mathématiques ${ }^{40}$. Là encore, une information couvre plusieurs acteurs et activités.

\begin{tabular}{|c|c|}
\hline Disciplines & Nombre d'enseignants \\
\hline arithmétique + calculs d'héritage & 49 \\
\hline science du temps & 11 \\
\hline astrologie & 0 \\
\hline géométrie & 7 \\
\hline théorie planétaire & 4 \\
\hline algèbre & 8 \\
\hline manuels d'astronomie & 4 \\
\hline éphémérides & 2 \\
\hline instruments & 3 \\
\hline arpentage & 5 \\
\hline
\end{tabular}

Tableau 2 : Nombre d'enseignants par discipline mentionnés dans le dictionnaire biographique de Shams al-Dīn al-Sakhāwī.

Des données similaires, quoique moins détaillées, peuvent être trouvées dans d'autres dictionnaires biographiques et dans des chroniques historiques, en particulier celles écrites sur les sociétés islamiques dont la langue de culture était le persan. Leur exploration et leur analyse approfondie augmenteraient nos connaissances de l'enseignement des mathématiques à différentes périodes et dans différentes régions.

Ma contribution avait ici deux objectifs. Tout d'abord, j'ai voulu expliquer pourquoi nous en savons si peu sur la façon dont les mathématiques et les sciences exactes ont été enseignées et étudiées dans les sociétés islamiques jusqu'en 1500. Deuxièmement, j'ai voulu réfléchir aux problèmes méthodologiques que nous rencontrons lorsque nous souhaitons étudier l'histoire de l'enseignement des mathématiques dans de nombreuses sociétés islamiques différentes et j'ai voulu indiquer qu'il existe d'autres sources que les textes scolaires eux-mêmes.

Les deux principaux obstacles à la production d'études solides et fiables sur les questions d'éducation sont l'ensemble des préjugés politiques, idéologiques, historiographiques et scientifiques qui prévalent parmi les historiens des mathématiques et des sciences exactes dans les

39. Ibid., vol. 1, p. 228.

40. Ibid., vol. 1, p. 126. 
sociétés islamiques et la préférence accordée aux programmes de recherche qui découlent de ces préjugés. Si l'arsenal méthodologique exploité et maîtrisé par les historiens des mathématiques et des sciences apparentées leur a permis d'étudier les contenus de ces disciplines, c'est moins le cas pour leurs contextes. Il a constitué au contraire un obstacle important, auquel on peut ajouter la production inégale de sources historiques dans les différentes régions du monde islamique et les différences que présentent leurs caractéristiques et leurs propriétés.

Surmonter ces obstacles, c'est rompre avec les programmes de recherche dominants, apprendre de nouvelles méthodes et trouver de nouvelles sources. Les dictionnaires biographiques et les éléments paratextuels se sont avérésêtre de nouveaux types de sources particulièrement féconds lorsqu'on les étudie systématiquement. C'est pourquoi j'ai mis ici l'accent sur leur présentation et sur leur potentialité particulière.

Compte tenu de l'espace limité dont je dispose dans cette contribution, je n'ai pas pu présenter un nombre suffisant d'exemples illustrant les multiples résultats que l'on peut obtenir en étudiant les textes scolaires mathématiques : leurs caractéristiques structurelles, visuelles, figurées et logiques (titre ; table des matières ; divisions en différents types de parties ; références ; diagrammes ; tableaux ; images ; normalisation terminologique ; etc.), leurs modes d'enseignement et d'apprentissage, leurs associations avec d'autres types de textes, les genres auxquels ils appartiennent, leurs diffusions géographique et institutionnelle ou tout autre type de réseau dans lequel ils peuvent s'inscrire. Je n'ai donc pas pu développer de façon suffisamment détaillée ma vision actuelle de l'enseignement des mathématiques dans les sociétés islamiques d'Égypte, de Syrie, d'Anatolie, d'Iran et d'Afrique du Nord. Cette éducation était informelle dans la mesure où elle se concentrait sur les personnes et les textes. Ceci s'applique à l'ensemble de la période envisagée dans cet article. À partir du XII siècle environ, ce caractère informel s'est fixé du fait de l'institutionnalisation relativement récente des donations légales, des allocations, des provisions matérielles, de postes de professeurs et d'autres types de personnel. Il s'est stabilisé à travers des institutions sociales telles que le mariage, les voyages aux fins d'apprentissage, les pèlerinages, le mécénat de cour et les procédures bien établies pour enseigner et acquérir une réputation d'enseignant dans les disciplines religieuses. Le résultat de ce mélange a été une stabilité centenaire de l'enseignement des mathématiques sur une base toujours changeante d'autorités, de (parties de) textes, de prescriptions comportementales et d'objectifs.

Sonja Brentjes - Institut Max Planck d'histoire des sciences (Berlin) 
Pourquoi et comment étudier l'histoire de l'enseignement des mathématiques dans les sociétés islamiques entre 750 et 1500

Dans cet article, je cherche à poser un certain nombre de questions sur la manière de traiter l'énorme quantité de textes mathématiques élémentaires en arabe, en persan et en turc conservés dans différents types de bibliothèques au cours de nombreux siècles et à travers plusieurs pays. Je propose de les traiter principalement comme les témoins d'un système éducatif étendu qui a commencé à inclure les sciences mathématiques à partir du XII siècle et à s'étendre à de nombreuses régions, même en dehors du monde islamique classique et des grands centres urbains. Je propose un certain nombre de réflexions sur les raisons pour lesquelles ces textes ont été ignorés par les historiens des sciences dans les sociétés islamiques pendant environ un demi-siècle et sur la façon dont les objectifs et les méthodes de recherche appliqués par les plus grands historiens nous ont empêchés d'étudier un corpus aussi important ainsi que leur cadre institutionnel. Je prolonge ces questions historiographiques avec une série de propositions sur la manière de compléter l'étude des textes mathématiques afin de trouver des réponses à la question de savoir comment l'enseignement des sciences mathématiques était organisé dans un contexte principalement informel, comment sont apparus les canons d'ouvrages pédagogiques et quelles pratiques pédagogiques se sont développées au contact des disciplines religieuses qui dominaient les milieux éducatifs.

biographies, éducation, madrasa, mathématiques, paratexte, sociétés islamiques

Why and How to Study the History of Mathematical Education in Islamic Societies between 750 and 1500

In this paper, I will raise questions about how to tackle the vast number of elementary mathematical texts in Arabic, Persian and Ottoman Turkish, that have been preserved in different kinds of libraries over many centuries and countries. I consider them primarily as witnesses of a widespread educational system that began to include the mathematical sciences from the twelfth century onward and spread into many different regions, even outside the classical Islamic world and beyond the major urban centers. I offer reflections on why these texts have been overlooked by historians of science in Islamic societies for about half a century and how the research aims and methods applied by leading historians have prevented us from studying such a major corpus of texts as well as their institutional context. I continue these historiographical questions with proposals for how to complement the study of the mathematical texts in order to learn how the teaching of mathematical sciences was organized, in a primarily informal context, how teaching canons emerged, and which teaching practices developed in contact with religious disciplines that dominated the educational environment.

biographies, education, Islamic societies, mathematics, madrasa, paratext 
